\title{
Design and Development of a Real-Time Model Attitude Measurement System for Hypersonic Facilities
}

\author{
Thomas W. Jones ${ }^{*}$ and Charles B. Lunsford ${ }^{\dagger}$ \\ NASA Langley Research Center \\ Hampton, VA 23681-2199
}

\begin{abstract}
A series of wind tunnel tests have been conducted to evaluate a multi-camera videogrammetric system designed to measure model attitude in hypersonic facilities. The technique utilizes processed video data and applies photogrammetric principles for point tracking to compute model position including pitch, roll and yaw variables. A discussion of the constraints encountered during the design, development, and testing process, including lighting, vibration, operational range and optical access is included. Initial measurement results from the NASA Langley Research Center (LaRC) 31-Inch Mach 10 tunnel are presented.
\end{abstract}

\section{Introduction}

$\mathrm{T}$ HE LaRC 31-Inch Mach 10 tunnel has a closed 31 x 31 inch test section with a contoured three-dimensional water-cooled nozzle to provide a Mach number capability of $10 .^{1}$ A view of the facility and the model injection system is provided in Figure 1. A hydraulically operated, side-mounted model injection system injects the model into the flow after the flow stream has been preheated. The hydraulic injection/retraction support mechanism is capable of providing an angle-of-attack (AoA) range of $\pm 90^{\circ}$. The sideslip (Yaw) range of motion is $\pm 5^{\circ}$. The model support mechanism also includes the mechanical position feedback sensors that provide pitch and yaw measurements (denoted by $\mathrm{M}_{\mathrm{ss}}$ ). These sensors have good wind-off accuracy but fail to account for sting bending due to aerodynamic loads. Thus pitch, yaw, and roll may have bias errors, which are not improved with repeat measurements, that may compromise test objectives.

As is the case for most hypersonic wind tunnel facilities, the size of typical models tested excludes on-board instrumentation. While servo accelerometers are routinely used for angle-of-attack measurements in most largescale wind tunnel facilities, ${ }^{2}$ their size and packaging requirement has prevented their use in small hypersonic models. While sting loading conditions that effect model attitude can be estimated, the need for an independent measurement system to accurately determine this deflection and provide a more accurate feedback of the models true position is the driving force behind the effort reported here.

\section{Aerodynamic Testing Requirements}

Many advanced hypersonic concept configurations are tested near cruise in the 31 Inch tunnel over a very small angle of attack range near zero. However, some re-entry vehicle configurations require testing at a high angle of attack. At these conditions the associated flow physics are complex and may exhibit attached oblique shocks, detached bow shocks, large regions of separated chaotic flow, body generated vortices, and thick turbulent boundary layers, any of which may greatly influence model dynamics and the associated sting bending. ${ }^{3}$

These characteristics will be highly dependent on aerodynamic angle of attack, thus requiring a high fidelity model orientation measurement capability over a large AoA range. Current industry-government program goals call for a wind tunnel measurement angular resolution of 0.1 deg or better. ${ }^{4}$

\footnotetext{
* Aerospace Engineer, Advanced Sensing and Optical Measurement Branch, M/S 238, Member AIAA.

${ }^{\dagger}$ Aerospace Engineer, Advanced Sensing and Optical Measurement Branch, M/S 238.
} 


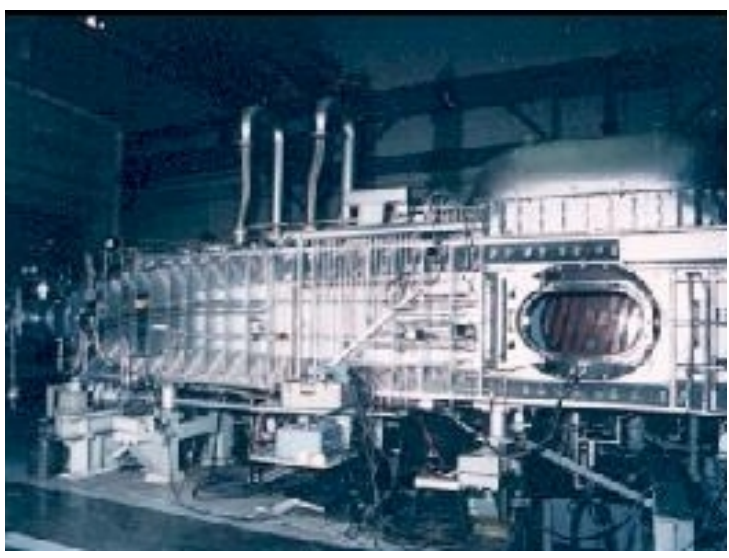

(a) 31-Inch Mach 10 Air Tunnel

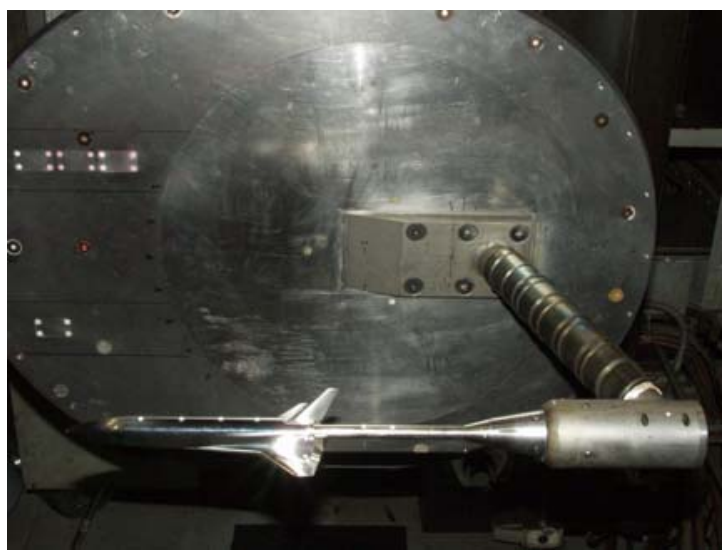

(b) Model injection system - 31-Inch Mach 10 Tunnel

Figure 1. NASA Langley 31-Inch Mach 10 hypersonic facility.

\section{System Design}

Recent modifications to the facility have now provided optical access to the test section from three of the possible four sides. This modification enables video based measurement systems $\left(\mathrm{V}_{\mathrm{AoA}}\right)$ to be considered as a leading candidate for providing model attitude data. To meet the aerodynamic test requirements a two-camera video system was designed that employed the basic principles of photogrammetry.

Photogrammetry is the science of measuring the location and size of three-dimensional (3D) objects with photographs. The data analysis procedures are related to those used in surveying. When dealing with time sequences of images, this technology is often called "videogrammetry" (or "videometrics") instead of "photogrammetry". High-contrast circular retroreflective targets are usually installed on the object to serve as discrete points when the highest measurement accuracies are required. These measured sets of object points can characterize shape or position. The images of the targeted points are processed to determine the 3D coordinates of the discrete points on the object's surface by triangulating the correspondence of intersecting light rays for the point. An example of a multi-camera configuration is depicted in Figure 2. Even though processing requirements for a photogrammetric solution can be intensive, the operating speed of modern PC workstations makes photogrammetric results possible in real time.

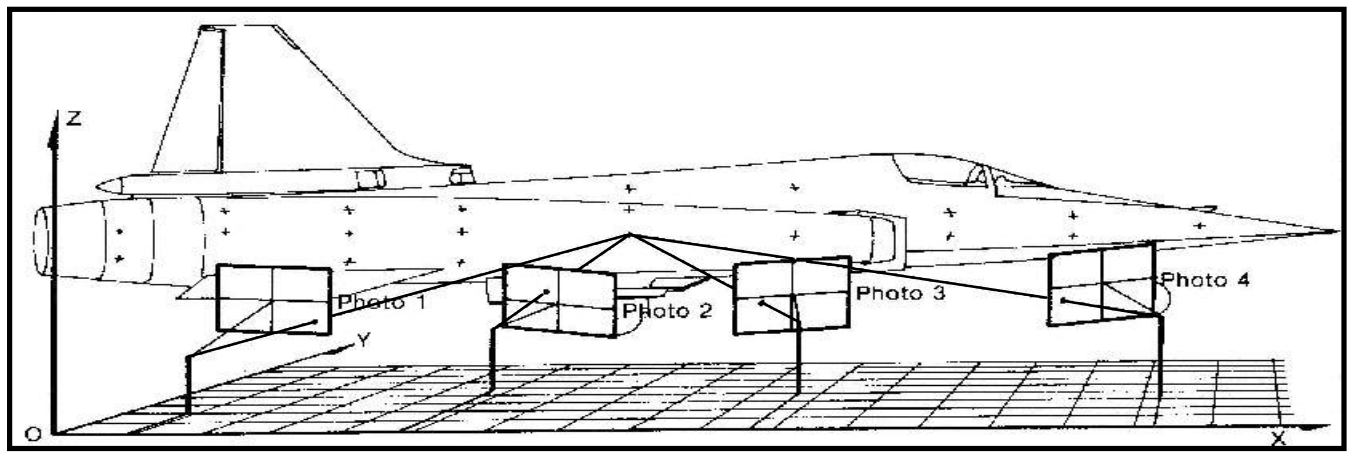

Figure 2. Photogrammetric triangulation principle of intersecting light rays for 3D point determination.

The camera specifications were partially derived from the maximum desirable target size. An assumption was made that the generally small surface area of the models tested in hypersonic facilities would restrict the size of surface targets to $2 \mathrm{~mm}$. Since the retroreflective targeting material has a surface thickness of 0.004 inches, an acrylic coating is applied to feather the edges of the targets to minimize the flow disturbance. To support high accuracy sub-pixel centroid determination a minimum diameter of 5 pixels on the image plane is needed from each of the targets. To accurately track the model through its range of motion a minimum of 5 targets are desired. The target size and the estimated working distance from the model dictated the need for a 1 mega-pixel class camera. 
The cameras selected were high-resolution Hitachi KP-F120's with 2/3 inch CCD sensor and $1392 \times 1040$ resolution. This camera enables reasonable centroid determination for $2 \mathrm{~mm}$ targets when viewed through a 12.5 $\mathrm{mm}$ lens at a distance of approximately 1 meter. The cameras are synchronized and provide data output rates (i.e. frame rates) as high as 30 hertz (Hz.). A diagram of the proposed camera arrangement using the tunnels top optical access window is provided in Figure 3.

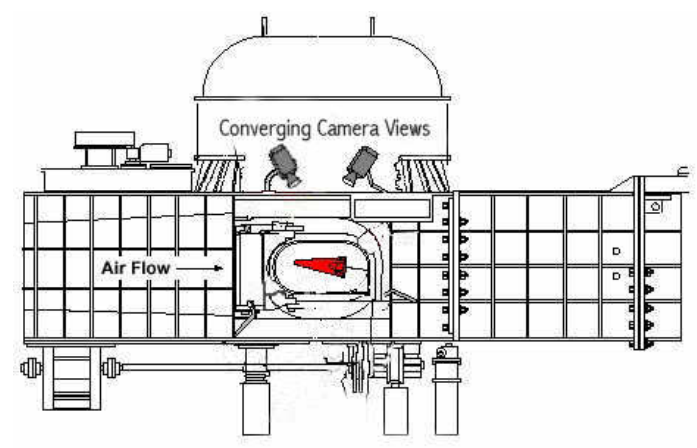

Figure 3. Camera-tunnel arrangement

Testing at the 31 Inch facility identified the best optical access point to achieve the maximum measurable pitch range using the fixed position two-camera setup. The position selected is forward facing with converging views to record the model position. In the configuration shown in Figure 4a the system is capable of tracking a model with surfaces parallel to the flow over a range of -20 to 40 degrees. The technique derives model position from an array of retroreflective targets on the model that are tracked throughout a recorded video sequence.

Figure 4b shows the view of a typical hypersonic model with attached retroreflective targets in the 31 inch tunnel test section with the video camera in the foreground. To achieve the highest accuracy the two-camera system should maintain a 60-90 degree convergence angle. However, the small access window on top of the test section restricted the camera separation to approximately 36 degrees.

To provide flexibility and portability the system is built on a T-slotted aluminum frame that allows the cameras to be quickly and easily repositioned. The aluminum frame supports a side mounted power/data distribution panel that regulates all of the power, control and data for the cameras and lighting, making the system completely self contained. To support the need for long cable lengths, fiber optic converters were added to convert the digital data from the cameras.

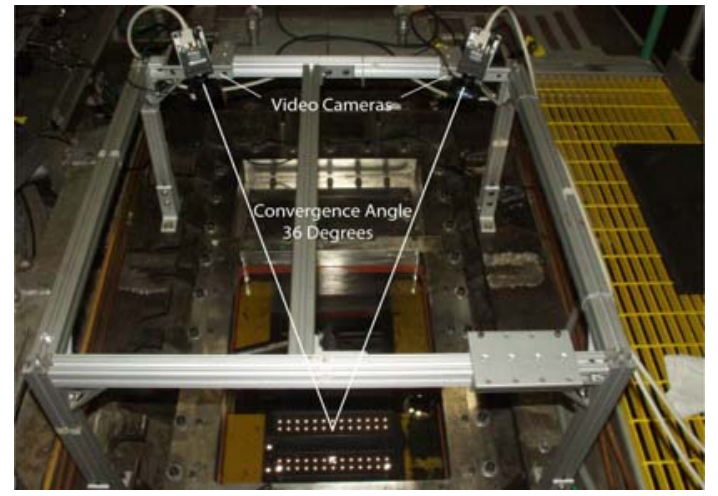

(a) Video camera convergent view configuration

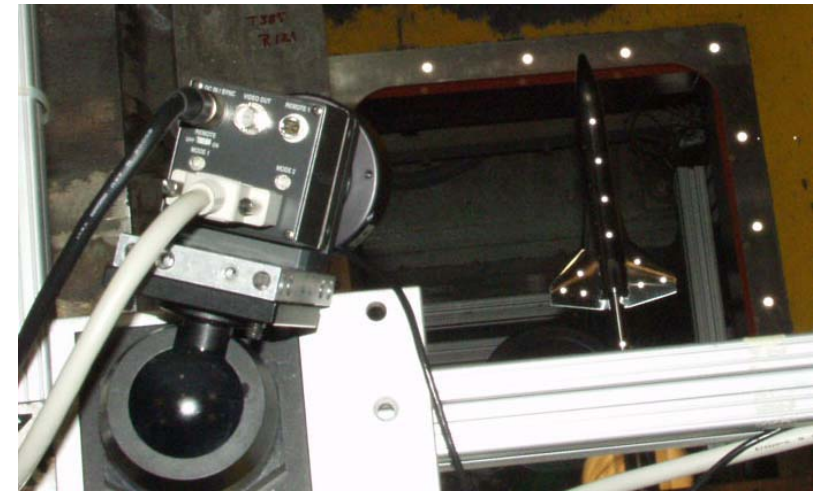

(b) Representative view of model in test section

Figure 4. Camera mechanical support hardware

\section{A. Processing Algorithm}

Photogrammetry offers several derivation methods for resolving camera parameters and object space coordinates. For a camera the relationship between object space point, perspective center and image point is described by the collinearity equations, ${ }^{5}$ the fundamental equations of photogrammetry. For our application a fourstep approach is followed. The first step is the pre-run calibration to determine the camera parameters, specifically the principle distance (focal length when the camera is focused on infinity), lens distortions, and perspective center. In the second step a technique known as "Resection" will be used to determine the camera position and orientation. Resection relies on an existing knowledge of the coordinates of multiple control points. For this case the coordinates of the control points, located on the floor of the test section, have been independently established to a high level of accuracy to minimize the potential bias error that may be introduced throughout the calculations. The camera orientation angles and location of the perspective center are referred to as the exterior orientation parameters. 
The third step in determining the object space coordinates of the targets is known as "Intersection". For this step it is assumed the exterior orientation parameters have been successfully determined through the second step of resection. For the intersection process, the object space co-ordinates of a target on the model can be determined by a least squares estimation of the collinearity equations.

The final step derives the models angular position by treating all targets on the model as a rigid body. The computed global coordinates of the targets are inputs to the solution. The models position is based on a three dimensional similarity transformation between the zero position, obtained as a wind-off zero point, and the measurements computed throughout the tracking process. A least squares estimation solution is used to reconcile redundant target coordinate data and appropriately process the statistical fluctuations of the measured target locations. ${ }^{6}$ The calculations include a full error propagation of the precisions of the tracked target coordinates into the solution and therefore provide precisions or uncertainty limits for pitch, roll and yaw. While most of the data presented here was post processed to insure accuracy, the algorithm is under development to support real time operations.

\section{B. Calibration}

As with any measurement system calibration is a primary concern. The general technique for camera calibration in a photogrammetric measurement system requires the recording of a series of images of a known target field from multiple perspectives. The images are then used to perform a camera calibration, determining the principal point location, principal distance, and lens distortions. In addition, the process establishes a coordinate system through the determination of the cameras relative orientation from which all measurements are based. However, in this case access to the tunnel test section area is limited due to safety issues. Therefore it was necessary to develop a calibration technique that didn't require removing the cameras and delaying tunnel operations each time a calibration is conducted. To meet this requirement a sting-mounted calibration plate with beveled edges was designed to provide the ability to perform in-situ calibrations, Figure 5 a. shows the plate in the test section of the 31inch hypersonic facility. The calibration plate is attached to the model support system in the same manner as a model, its embedded sting, visible in Figure 5b. provides the means to support easy installation and removal. The calibration plate is injected into the tunnel in a wind-off condition and the host computer then records images of the calibration plate at various pitch angles.

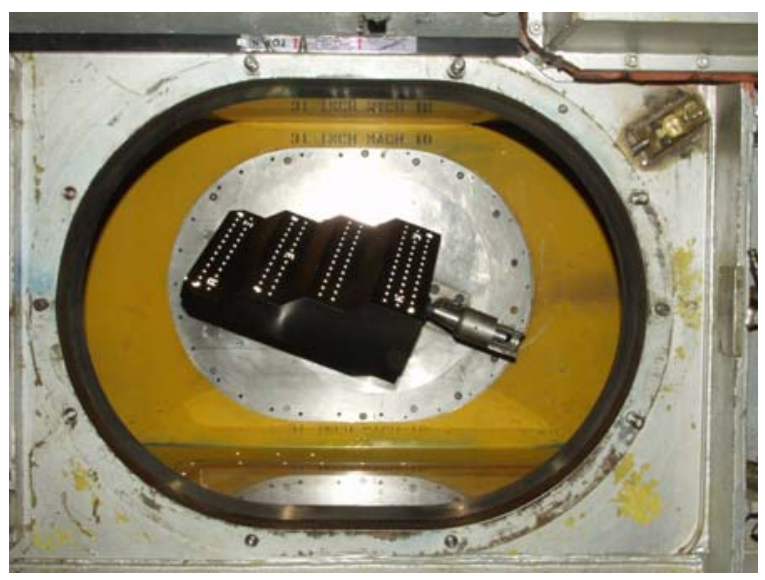

(a) Test section view sting-mounted calibation plate

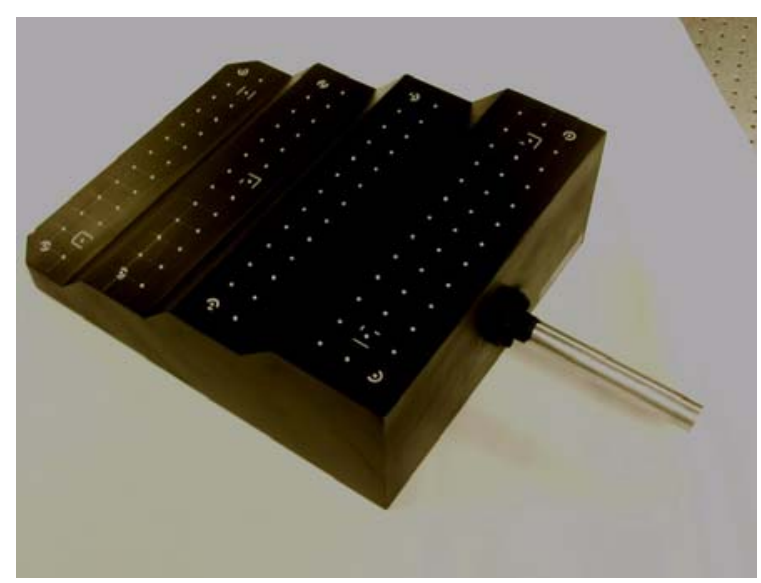

(b) Calibration plate with sting mounting adapter

Figure 5. Video camera calibration plate

\section{Lighting}

In most videogrammetric applications targets are illuminated with a standard white light source. However, phosphor thermography a color imaging based measurement system is routinely used in the Langley hypersonic facilities. ${ }^{7}$ The phosphor technique utilizes a Ultra-violet (UV) source that is absorbed by the phosphor coating on the model and emits a response in the visible range based on the thermal profile of the model. The videogrammetric system had to be designed to accommodate the phosphor technique without interfering with the data quality of that technique. 
The videogrammetric system was designed to prevent interference with the phosphor's source lighting (UV) and the measured response in the visible spectrum of the model during testing. Target illumination for the videogrammetric system was designed to operate in the near IR region $(860 \mathrm{~nm})$ using a commercially available lens mounted LED light ring to prevent interference from both ambient lighting and the phosphor thermography technique. To further enhance the contrast, optical filters were added to the lens to block all visible sources below $700 \mathrm{~nm}$. To evaluate the efficiency of the lighting system design a phosphor thermography test was conducted with both test techniques active during data recording. The cameras and lighting for the two techniques were positioned in opposite windows with the phosphor camera system viewing the model from the side window and the videogrammetry system viewing from the top test section window. A simultaneously recorded image of the model as viewed by each system is presented in Figure 6. There is no evidence of interference from either systems perspective.

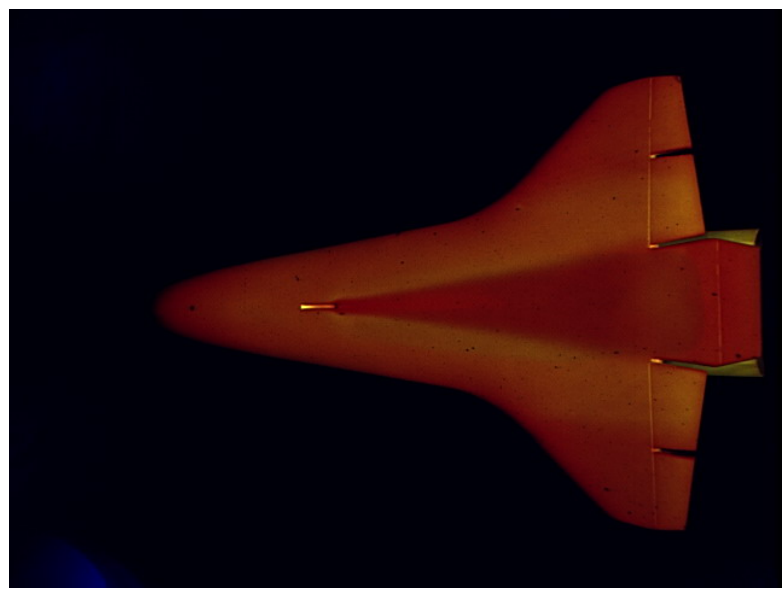

(a) UV illuminated model

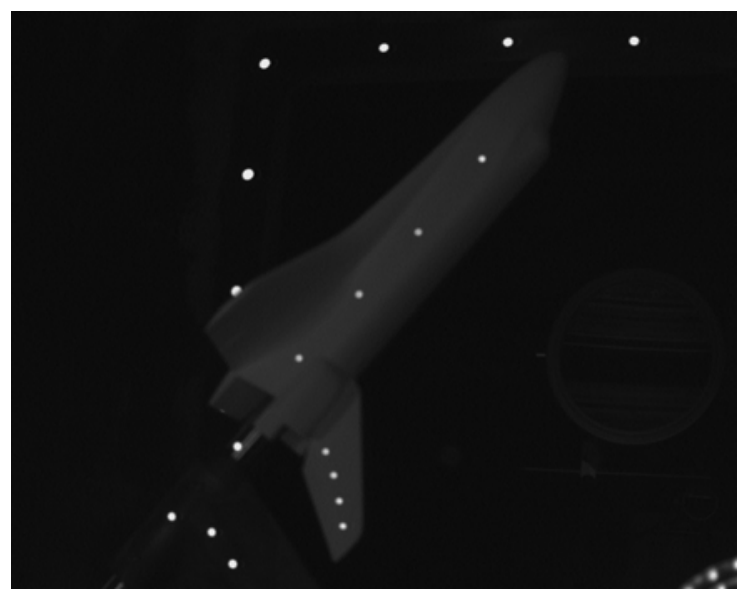

(b) IR illuminated targets for AoA tracking

Figure 6. Concurrently recorded images during Phosphor Thermography model testing.

While the phosphor technique represents a significant percentage of hypersonic facility testing, stainless steel models are used for an even greater percentage of tests. Stainless steel models have traditionally presented problems to image based measurement systems due to the reflections and glints from the surface causing false targets and occlusions. An example of a model image with significant target occlusions is shown in Figure 7(a). In general retroreflective targets reflect the light back to the source along the incident axis. In the case where the targets are attached to a reflective surface such as a polished steel model the model itself will reflect a majority of the light at an angle equal to the angle of incidence. In a two camera configuration where each camera has independent lighting and the separation angles from a perpendicular to the imaged surface are approximately equal there is a strong possibility of glints "washing-out" surface targets. To combat this effect a cross-polarization technique has been developed that greatly reduces the glints and reflections. The technique as illustrated in Figure 7(b) has a linear polarizing filter in front of each LED array and the camera. The polarizers are aligned such that the linear polarization of the LED lighting produced by each is crossed relative to each other. The polarization angle of the camera filter is aligned to match that of the attached LED light. The polarization angle of the camera filter blocks the light from the second cameras LED source that is polarized to an angle 90 degrees relative to the first camera. Results using this technique on models with relatively planer surfaces are encouraging; showing significant improvement in image contrast compared to cases with no polarization filters. Images from the same camera recorded with and without the polarization filters are shown in Figure 7(a) and (c). While the effectiveness of the technique is largely dependent on the model shape, most models tested in this class of facilities do not include complex geometries that may limit the effectiveness of the technique. Further studies of this technique are underway. ${ }^{8}$ 


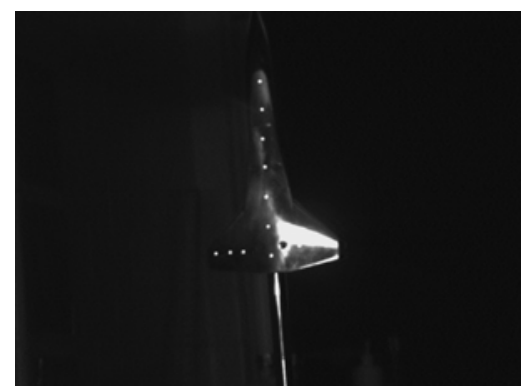

(a) Image without polarizer

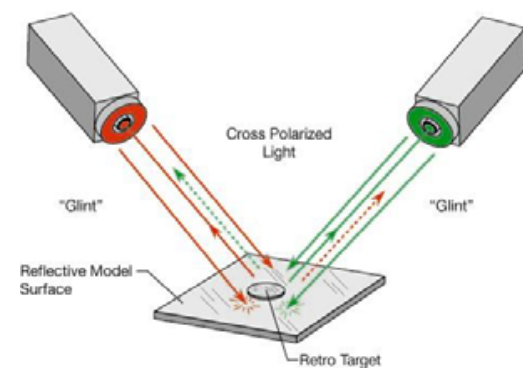

(b) Two camera cross polarization

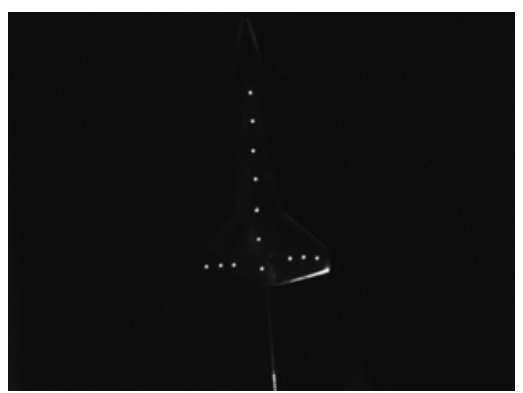

(c) Image recorded with x-polarizer

Figure 7. New cross polarization technique for glint removal

\section{Vibration}

Since the proposed configuration requires the cameras to be rigidly mounted to the tunnel shell, the effects of tunnel vibration on the measurement precision were investigated. To evaluate the precision of the image based measurements and to characterize tunnel vibrations sensed by the cameras, a group of seven control points both in the tunnel test section and on the external tunnel structure were measured. The control points on the test section floor were visible to both cameras as shown in Figure 4(b). To fairly evaluate the vibration induced by the tunnel operating at high Mach number the measurements were recorded before, during and after the tunnel run. To subject the cameras to the highest possible vibration a tunnel run was conducted with a stagnation pressure of $1450 \mathrm{psi}$ and the model at a pitch angle of 20 degrees. The control points were recorded at $30 \mathrm{~Hz}$. for the full 80 seconds of the tunnel run. Approximately 2500 points were recorded and analyzed for each control point. The points were analyzed based on their 3 dimensional XYZ position. Representative statistical results are presented in Table 1. As shown there is no significant shift in the control points position attributable to tunnel or camera vibration. Similar results were found when the cameras were repositioned to record a point not on or attached to the tunnel structure. It should be noted the coordinate system convention is non-standard, with the $\mathrm{Y}$ axis in the direction of the air flow, the $\mathrm{X}$ axis to the left and the $\mathrm{Z}$ axis up. With this coordinate convention the lowest precision will be in the $\mathrm{Z}$ axis direction due to the small separation angle between the two cameras.

\begin{tabular}{llll}
\hline Control Point \# 1001 & X (inches) & Y (inches) & $Z$ Z (inches) \\
Minimum: & 10.628 & -6.093 & -1.0362 \\
Median: & 10.6309 & -6.0882 & -1.0255 \\
Maximum: & 10.6338 & -6.0833 & -1.0173 \\
& & & \\
Midrange: & 10.6309 & -6.08815 & -1.02675 \\
Range: & 0.0058 & 0.0096 & 0.0189 \\
Interquartile Range: & 0.00128 & 0.0017 & 0.0037 \\
Median Abs. Deviation: & 0.0006 & & \\
Mean: & 10.630 & -6.088 & -1.025 \\
Standard Deviation: & 0.000814 & 0.001351 & 0.0027910 \\
Variance: & $6.627 \mathrm{E}-007$ & $1.8251 \mathrm{E}-006$ & $7.790 \mathrm{E}-006$ \\
\hline
\end{tabular}

Table 1. Statistical analysis of fixed test section control point

\section{Integration and Testing}

At this stage in the development the goal is to benchmark the performance and identify any critical problems and deficiencies. Future testing will be used to fully characterize uncertainty and account for bias errors that are very difficult to determine under flow conditions without independent validation. This evaluation included two wind-on tests in the 31 Inch wind tunnel and the calibration laboratory using a typical polished steel hypersonic model. Angular measurements derived from 12 retroreflective targets tracked throughout the range of motion were compared to angles generated by the model support system sensors. 
The comparisons were based on a pitch sweep over a range of -4 degrees to +10 degrees. The results, shown in Figure 8 as a function of angle-of-attack, represent a difference between the computed pitch angle and that measured by the model support system $\left(\mathrm{V}_{\mathrm{AoA}}-\mathrm{M}_{\mathrm{ss}}\right)$. The results represent two data sets recorded over a three-day period. Each of the data sets represents an 80 second tunnel run. The tunnel conditions were held at a stagnation pressure of $1450 \mathrm{psi}$ and a stagnation temperature of 1250 degrees F. The model position for each recorded point was maintained for approximately 2 seconds during the pitch sweep. The outliers shown in the plots of Figure 8 are attributed to points recorded while the model was in motion. For a difference analysis, if the differences are scattered randomly about a mean of zero, then it could be concluded that there is no difference in the mean performance of the two systems. A pre-run wind-off data point indicated a 0.12 degree bias between the two systems at a 0 degree angle of attack. However there is a larger bias between the two measurements as a function of pitch angle that may represent a bias error and/or a sting bending effect due to aerodynamic loads. The two data sets show a mean difference of -0.3 degrees in Pitch at an angle of attack of -4.0 degrees. At angles approaching zero the difference is closer to zero. The discrepancy in the difference between the two data sets for positive angles of attack is currently the study of further evaluations.
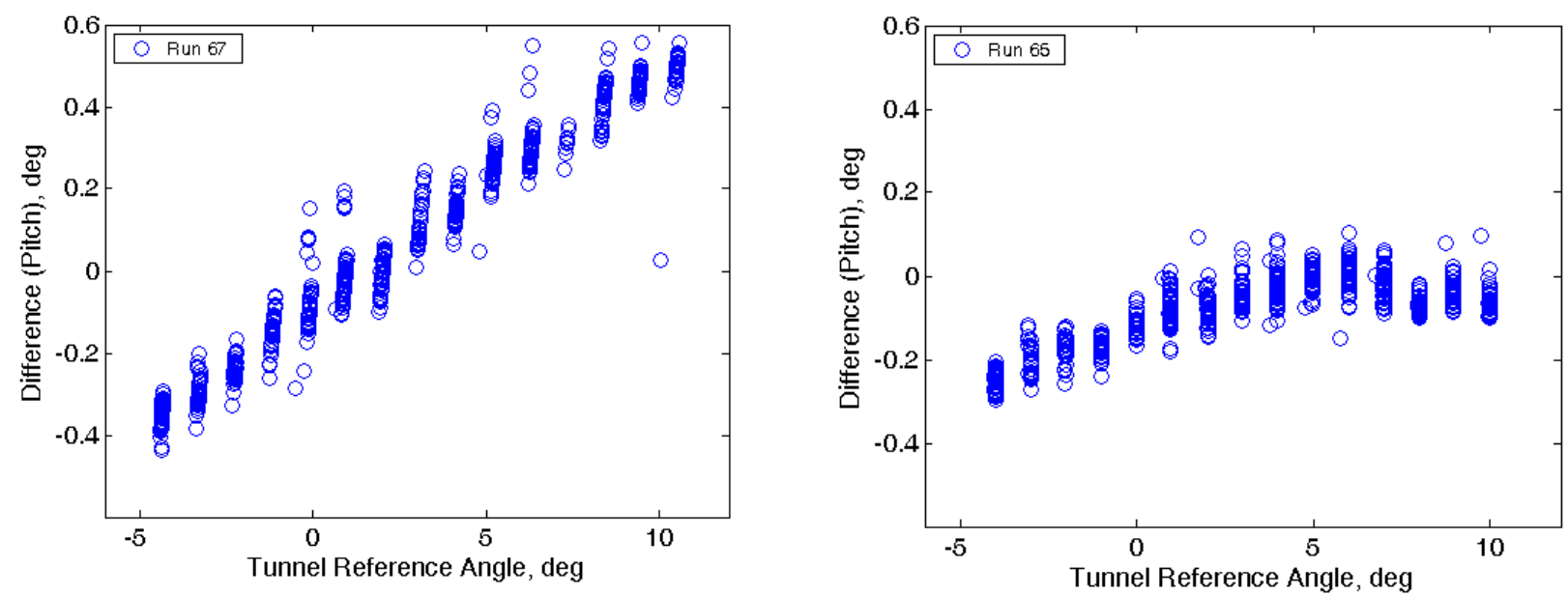

Figure 8. “Wind-on” tunnel data, difference between video AoA and model support system sensors

To investigate the difference between to the two runs with the available data we evaluated the rigid body transformation used to determine the 3D position on a point-by-point basis. A Unit Weight Estimate (UWE) was calculated for each point and plotted in Figure 9. The UWE is sigma zero for the least squares estimation solution to the 3D transformation. It measures the "goodness of fit" with larger numbers indicating a poorer result. The analysis indicated a very good point-to-point transformation for both of the runs, with "Run65" indicating a slight divergence for angles greater than zero. Inspection of the standard deviations for the computed pitch angle between the two data sets indicates a divergence that requires further investigation. It is assumed that this larger error band accounts for the divergence in the two difference plots, Figure 8. To accurately investigate the true bias that exist between the two measurement systems a series of "wind-off" pitch sweeps in the tunnel will need to be conducted with the support of an independent measurement system. This test is being planned as part of the on-going test and integration effort. 


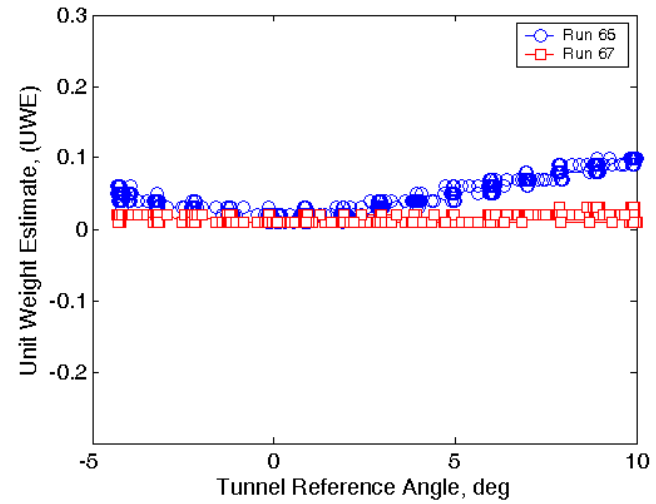

(a) Unit weight estimate of 3D tracking

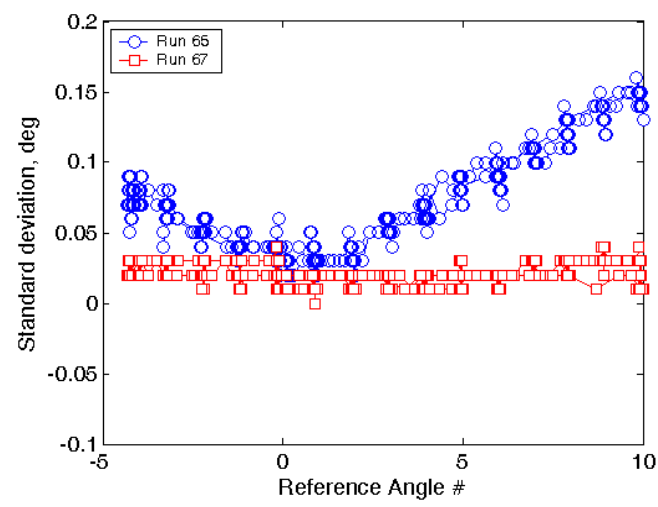

(b) Standard deviation of computed Pitch

Figure 9. Tunnel run comparisons of rigid body 3D angular measurement calculations

To accurately assess angular measurement capability in a controlled laboratory environment, the cameras were moved intact from the tunnel to the laboratory maintaining their camera/model geometry. The laboratory setup is

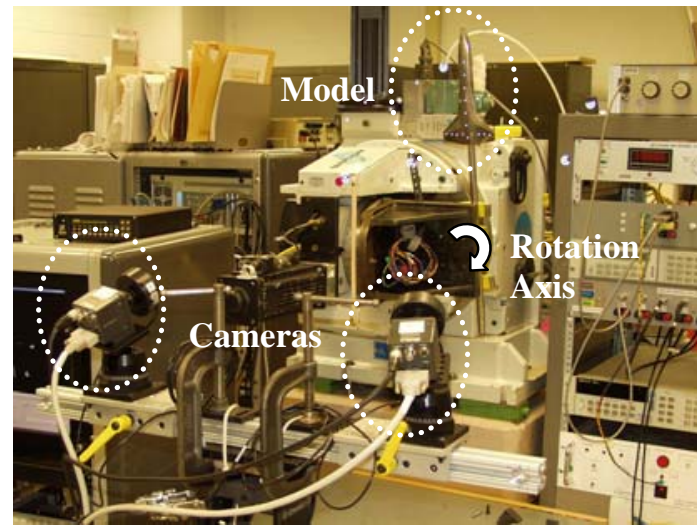

Figure 10. Laboratory setup of Optical AoA shown in Figure 10. An automatic two-axis angle indexer provided the laboratory measurement validation.

The indexer, used as a calibration standard at NASA LaRC, can be set in increments of 1 degree with an accuracy of 1 arc second on each axis. While the range of motion for the tunnel test was limited due to the safety limits for the strain-gauge balance, the lab tests were conducted over a 30 degree range (10 to 20) to demonstrate the capabilities over the designed measurement range. The model was positioned on the indexer with an axis of rotation that simulated the pitch motion relative to the cameras in the tunnel test. A series of five tests were conducted. Three of the tests represented "pitch only" sweeps, the last two included a 5 degree offset angle in yaw. A data point was taken at each 1 degree increment over the 30 degree range. To evaluate the repeatability and bias error of the data a least-squares regression analysis was performed with each of

the three "pitch only" sweeps combined into one dataset. The computed pitch, roll and yaw are fit with a zero-order polynomial least-squares curve. A confidence interval or prediction interval, at 95\% confidence level, can be computed. The confidence interval is the interval within which there is $95 \%$ certainty that the true mean lies. This confidence interval will become a bias that accompanies this curve fit. The prediction interval is an interval within which future data points are expected to lie $95 \%$ of the time ${ }^{9}$. Since the indexer is being used as the baseline measurement, examining the difference between its angle of attack reading and the video system will provide a measure of the uncertainty of the video derived angle-of-attack in a laboratory environment. The difference between the photogrammetric data and the indexer for the "pitch only" sweeps are plotted in Figure 11.

The derived data for model position in the laboratory indicates the bias error in target tracking for pitch is small and close to the desirable 0.1 deg band. The divergence that occurs between pitch and yaw in addition to the offset and wider scatter in the roll data are aspects that require further investigation to adequately resolve. Possible errors in the coordinate transformation process may contribute to the problem. The analysis of the laboratory results implies the best case by which we can gage the performance of the wind tunnel data. 

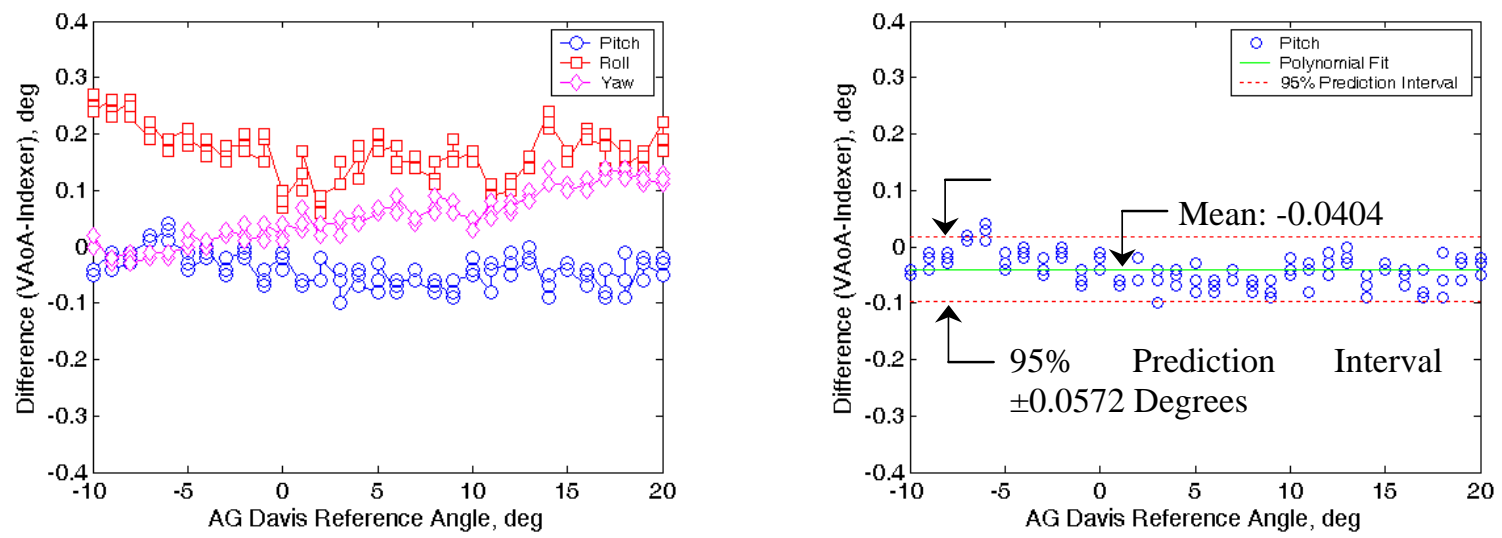

Figure 11. Laboratory test conducted with high accuracy indexer.

\section{Conclusions}

The investigation and development of an angular measurement system for hypersonic wind tunnel facilities located at NASA LaRC has been presented. The utilization of mega-pixel type video cameras and photogrammetric processing techniques in a prototype demonstration was addressed. All major aspects of the system design have been investigated. Camera calibration, lighting, data acquisition, target tracking, and model attitude derivations were explored and investigated to a level that confirmed acceptable performance. The operational capability was tested and demonstrated in wind tunnel and laboratory conditions.

The angular measurement performance based on the wind tunnel data was inconclusive. The disagreement in the data sets along with the unknown effects of aerodynamic loading prevent conclusive assessment of the performance in meeting the design requirement of 0.1 degree over the desired angle of attack range. However, the results of tests conducted in the laboratory using a high accuracy indexer were repeatable and consistent with expected performance.

While the angular measurement performance of 0.1 degree in pitch over an angle of attack range of 30 degrees was confirmed as part of the laboratory test. To better understand the systems performance in the wind tunnel a series of test with an independent validation are needed. The test will need to include a full characterization of the bias between the systems over the entire measurement range without air flow. The laboratory and tunnel wind-off determination of error along with test-to-test repeatability can then be used as a gauge for the angle of attack measurements with flow.

Various constraints including vibration, lighting, optical access and calibration associated with the system design were investigated and described in this report. The vibration analysis indicated the tunnel attached hardware configuration was stable and suitable for a multi-camera image based measurement system. Observations of fixed control points indicated measurement stability to 0.003 inches.

An innovative lighting technique using cross-polarization was demonstrated to substantially reduce glints and unwanted reflections for dual camera configurations. The techniques near IR operational spectrum does not interfere with other techniques such as phosphor thermography and should be adaptable to other facilities.

Optical access to the tunnel test section was gained through a top view window. This perspective allows target tracking over a large angle of attack range. The choice of locating the system on top of the test section may yield the weakest results in the pitch axis, a by product of the small separation (convergence) angle between the two cameras, but preliminary indications imply the effect on measurement accuracy is not significant. Improvements in the measurement accuracy in the pitch axis could be made by locating the system at the side window port, but at a substantial cost to tunnel access, since the side window/door port provides the primary test section access.

To minimize impact on tunnel operations an in-situ calibration technique using a sting mounted 3D calibration plate was developed. The calibration plate will allow routine calibration of the cameras without requiring special test section access.

Although the actual system has not been fully integrated into the Langley 31-Inch facility, the functional performance of the system in the facility demonstrates the systems capabilities. The system design is non-contact and non-intrusive in terms of the model and support system, only requiring a few $2 \mathrm{~mm}$ retroreflective targets. System calibrations are typically required only once per test. The portable self-contained nature of the systems design will allow the systems to be easily retrofitted to other hypersonic wind tunnel facilities at NASA LaRC. 
While the results presented here represent an interim report it appears that mega-pixel type video cameras and photogrammetric processing techniques are capable of satisfying current model orientation accuracy objectives.

1 J. R. Micol, Langley Aerothermodynamics Facilities Complex: Enhancements and Testing Capabilities , 36th AIAA Aerospace Sciences Meeting and Exhibit, Reno, Nevada, AIAA 98-0147, January 12-15, 1998, (4MB).

2 T.Finley and P. Tcheng, "Model Attitude Measurements at NASA Langley Research Center”, 30th Aerospace Sciences Meeting \& Exhibit, January 1992, Reno, NV, AIAA 92-0763.

3 B.Newman and Si-bok Yu, "Development of a High Accuracy Angular Measurement System for Langley Research Center Hypersonic Wind Tunnel Facilities,” NASA/CR-2003-212401, Langley Research Center Hampton, Virginia, August 2003.

4 Tripp, J.S., Tcheng, P., Burner, A.W., and Finley, T.D., "Summary Report of the First International Symposium on Strain Gauge Balances and Workshop on AoA/Model Deformation Measurement Techniques,” NASA-CP-1999-209101, Langley Research Center Hampton, Virginia, March, 1999.

5 K.B. Atkinson, Close Range Photogrammetry and Machine Vision, Whittles Publishing, Scotland, UK, 1996, pp. 24-27.

6 W.L.Snow, M.R. Shortis, “A Rigid Body Motion Analysis System for Offline Processing of Time Coded Video Sequences”, Videometrics IV, SPIE Proceedings Vol 2598, Oct 1995.

7 N. Ronald Merski, Reduction and Analysis of Phosphor Thermography Data With the IHEAT Software Package , 36th AIAA Aerospace Sciences Meeting and Exhibit, Reno, Nevada, AIAA 98-0712, January 12-15, 1998, pp. 21, (3MB).

8 Wells, J.M., Jones, T.W., “Polarization techniques applied to enhance photogrammetric measurements of reflective surfaces,” 46th AIAA/ASME/ASCE/AHS/ASC Structures, Structural Dynamics\& Materials Conference, AIAA-2005-1887, Austin, Texas, April 2005.

9 Montgomery, D.C. and Peck, E.A., Introduction to Linear Regression Analysis, John Wiley and Sons, New York, New York, 1982. 